The research in this paper was performed in connection with consulting agreements between the author and Continental Oil Co., Ponca City, Oklahoma, and Curtiss-Wright Aircraft Co., Wright Aeronautical Division, Woodridge, N. J. Curtiss-Wright provided all computer time used.

${ }^{1}$ L. F. RICHARDSON, "The approximate arithmetical solution by finite differences of physical problems involving differential equations with an application to stresses in a masonry dam," Roy. Soc., London, Phil. Trans., Sec. A, v. 210, 1910, p. 307-357.

2 D. Young, "Iterative methods for solving partial difference equations of elliptic type," Amer. Math. Soc., Trans., v. 76, 1954, p. 92-111.

${ }^{3}$ S. P. Frankel, "Convergence rates of iterative treatments of partial differential equations," $M T A C$, v. 4, 1950, p. 65-75.

"J. D. RILEY, "Iteration procedures for the Dirichlet difference problem," $M T A C, \mathrm{v} .8,1954$, p. 125-131.

' G. SHORTLEy, "Use of Tchebycheff polynomial operators in the numerical solution of boundary value problems," Jn. Appl. Phys., v. 24, 1953, p. 392-396.

$6 \mathrm{D}$. Young, "On Richardson's method for solving linear systems with positive definite matrices," Jn. Math. and Physics, v. 32, 1954, p. 243-255.

${ }^{7}$ D. YounG, op. cit., reference 2, p. 93.

${ }^{8}$ D. Young, op. cit., p. 108.

${ }^{9} \mathrm{D}$. Young, op. cit., section 4.

10 D. Young, op. cit., p. 96.

1 A. C. Artken, "On the iterative solution of a system of linear equations," Roy. Soc., Edinburgh, Proc., Sec. A, 63, 1950, p. 52-60.

${ }_{12}$ D. Young, op. cit., reference 2, p. 103.

13 The International Business Machines Company Type 701 Electronic Data Processing Machines located at the IBM Scientific Computing Service, 590 Madison Ave., New York City, N. Y.

i4 John Sheldon \& L. H. Thomas, “The use of large scale computing in physics," Jn. Appl. Phys., v. 24, 1953, p. 235-242.

\title{
Solutions of a Nonlinear Differential Equation Arising in the Theory of Diffusion Flames
}

An extended theory of diffusion flames [1] leads to a nonlinear second order differential equation, which is, in its non-dimensional form [2]:

$$
v^{\prime \prime}=\frac{K}{x+x_{1}} \cdot v \cdot\left(v+x+x_{1}-1\right)
$$

with the following two-point boundary conditions:

$$
v=1-x_{1} \text { at } x=0
$$

and

$$
v \rightarrow 0 \text { as } x \rightarrow \infty \text {. }
$$

This equation describes the reaction of sodium vapor diffusing into a halide, where $1-x_{1}$ and $K$ correspond effectively to a measure of the nozzle opening and the chemical rate of reaction, respectively. The equation has been solved numerically, with the aid of FERUT, to cover a wide range of experimental conditions. It presented many difficulties of a practical nature, which could hardly have been foreseen until considerable work had been done.

First of all, solutions for two cases, corresponding directly to specific experiments, were carried out by hand, using the equation in its original form [1] and employing MILNE's method of integration. Each of these required nearly a month of desk work by a proficient computer. One of them served as an independent check for the FERUT programs.

In order to adapt the problem for automatic solution it was decided to work 
over a mesh of parametric values of $K$ and $x_{1}$. Correspondence with experiment could then be obtained by interpolation into these results.

In an attempt to draw up a comprehensive program, certain difficulties soon became evident. For instance, because of the large variation in the parameters, the functions involved extended over a wide range of magnitudes, and in a manner

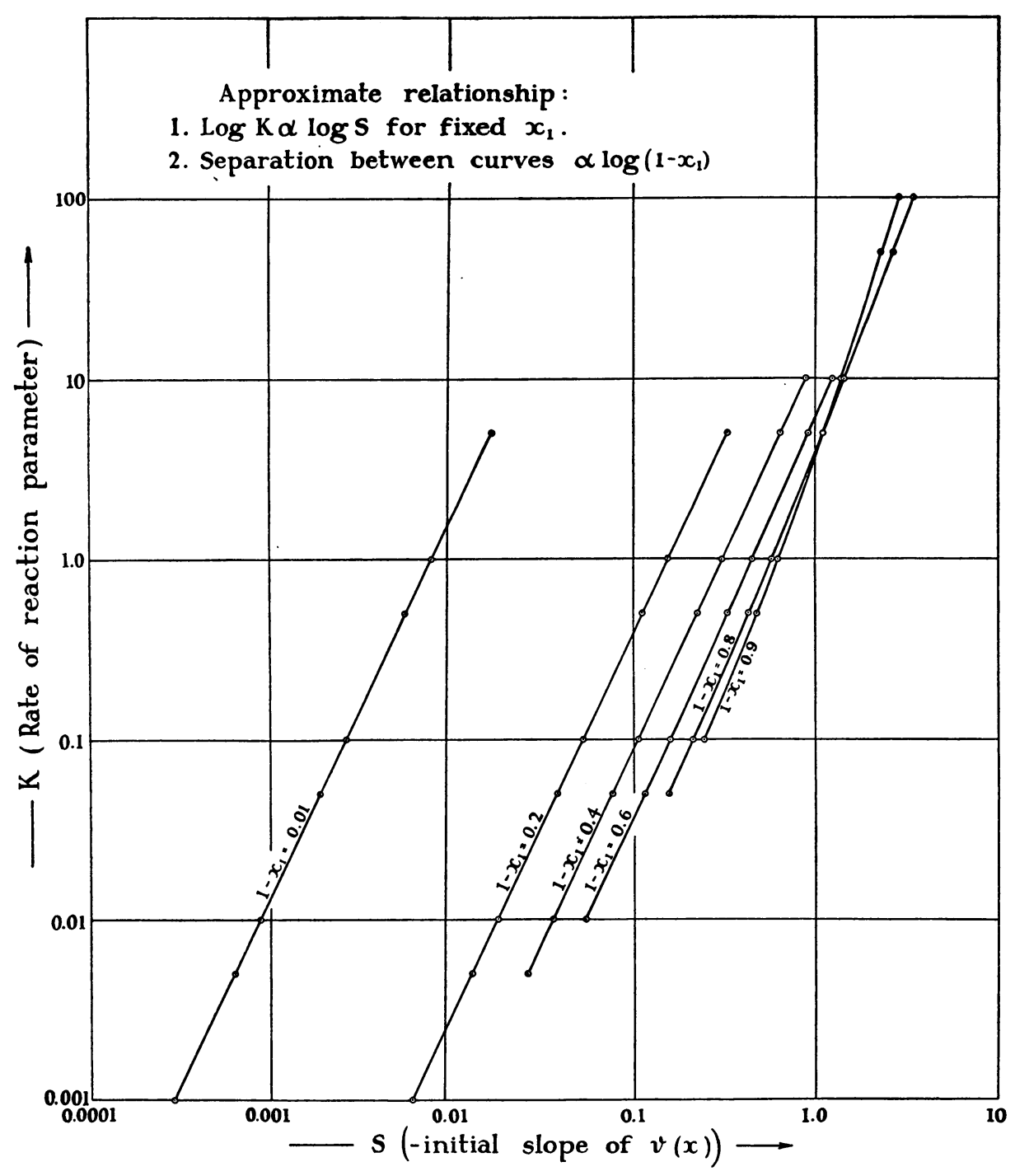

FIG. 1.

which could only be predicted from numerous and prohibitively tedious hand calculations. Also the effective value of $\infty$ was not known, but soon appeared to be significantly dependent on the combination of parameters used. This rendered impracticable the technique of integrating both outward from $x=0$ and inward from $x$ large. It was also noted that the solution was only defined for a particular 
value of the initial slope $v^{\prime}(0)$. This necessitated an iterative or repetitive numerical method, which at once introduced questions of convergence.

It was decided to use the Runge-Kutta process of integration as modified by GILL [3], a routine for this already being available in library form. This method does not require the separate calculation of starting values and cuts inherited errors to a minimum. However, it is slow in comparison with other methods [5]. The technique of repeated outwards integration, with systematic adjustment of initial slope to satisfy conditions at $\infty$, was adopted.

A fixed-point program, supplemented by a plotting routine to display on paper the shape of the approximating solutions, was first prepared. The method of adjustable pre-scaling [4] was employed. Scale factors were provided for $x_{1}$ and $K$, and it was hoped that these could always be selected to prevent numerical overflow and still give the desired accuracy. This program was used with some

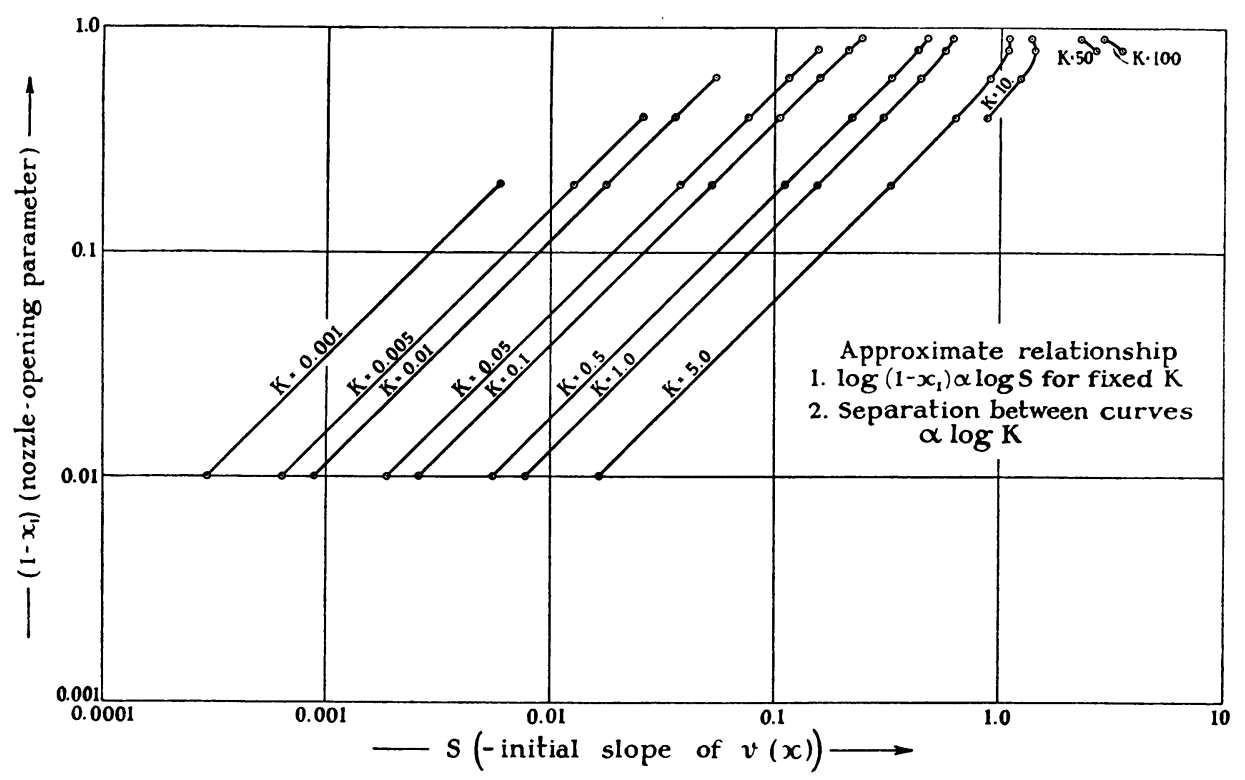

FIG. 2.

success for the smaller values of $1-x_{1}$ and $K$, but proved inadequate elsewhere. Either overflow occurred regardless of choice of scaling factors or the interval of integration could not be suitably refined. Sufficient work was done to reveal that the entire solution, and not just its terminal behavior, was very sensitive to slight changes in the initial slope. This effect became progressively more pronounced towards the larger values of $K$ and $1-x_{1}$ and made it necessary to recommence each integration at $x=0$. Also, the converged value of the initial slope varied considerably from one parametric case to the next. Numerical details to supplement these various statements are summarized below.

In view of these many practical difficulties, it was decided to make a direct attack on the problem with a floating-point program. This was actually written in Transcode, a system of automatic coding recently developed for FERUT. It paralleled the fixed-point program with the exclusion of the plotting routine, and 
proved much easier to develop and operate. The reduction in running speed by a factor six was not intolerable.

Some operating details follow : The initial slope, $v^{\prime}(0)$, was defined as $-s$, since it was always found to be negative. Preliminary runs for each parametric case were made as follows:

1. To determine the interval size which would yield the value of $v(x)$ to between 3 and 5 significant figures.

2. To determine two values of the trial $s$, one producing divergence to $+\infty$ and the other divergence to $-\infty$.

3. To determine the effective value of $x \rightarrow \infty$ for applying the test for divergence.

Final runs for each parametric case were made to determine $s$ such that $v(x)$ dropped to within $1 / 200$ of its initial value and remained parallel to the $x$ axis for at least $1 / 20$ of the total range of integration. The value of $x$ at which $v(x)$ first reached $1 / 200$ of its initial value was defined as the effective value of $\infty$.

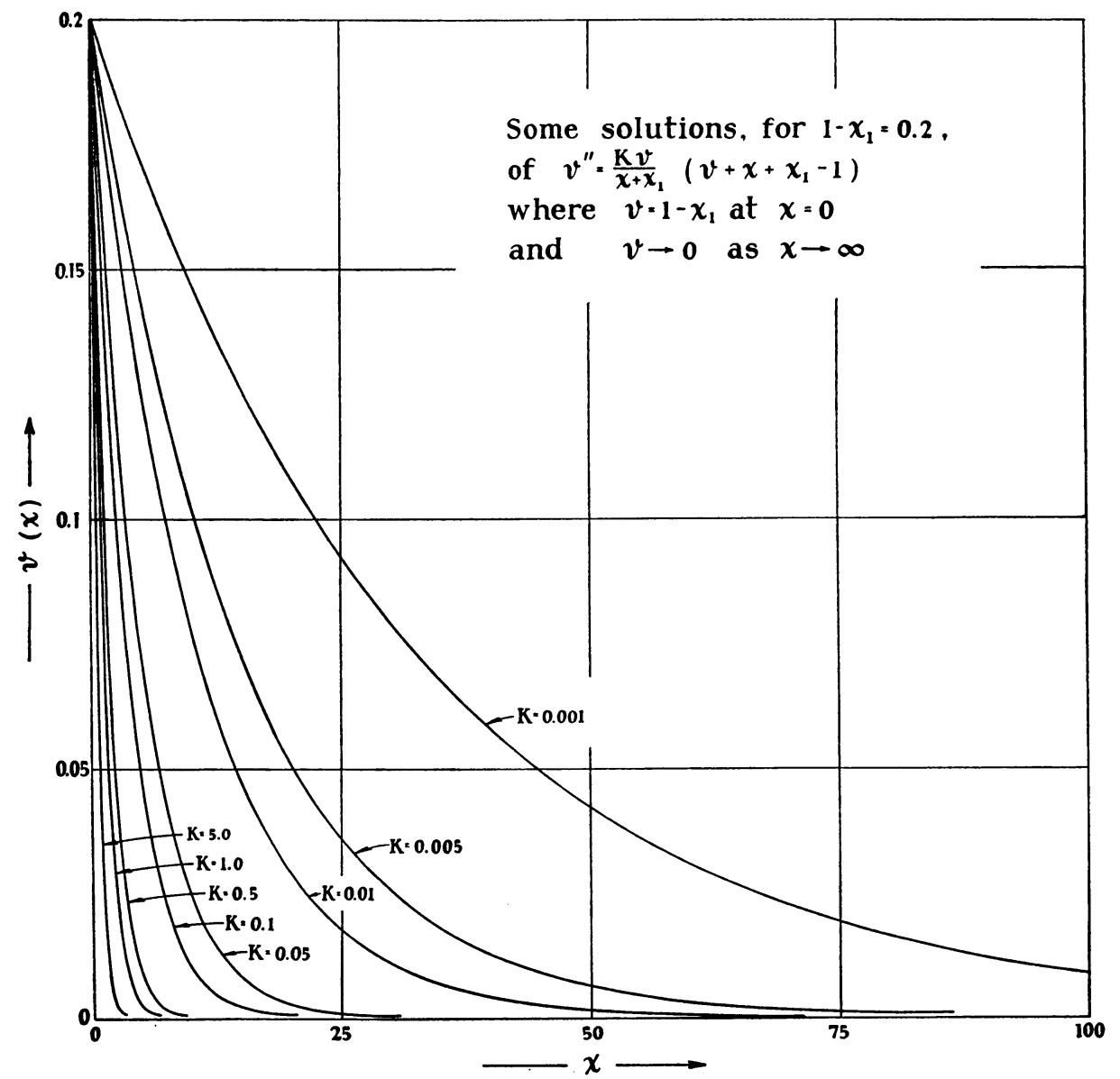

Fig. 3 . 
In the fixed-point program, $s$ was automatically adjusted by averaging the two most recent trial values known to produce divergence in either direction. This proved rather crude in view of the sensitivity of the solution to the trial value of $s$. With the floating-point program, adjustment of $s$ was made by higher order interpolation away from the computer. Since each run over the range of integration required from 5 to 15 minutes, this procedure could be followed quite efficiently. Preliminary runs required integration over about 20 to 50 steps. However, as many as 180 steps were necessary in some of the final runs, when the value of $s$ was approaching convergence. Also, the interval size could not be varied suitably over the range in view of the extremely divergent behavior of the approximating solutions.

Figures 1 and 2 illustrate the relationships discovered between the converged $s$ and each of $K$ and $1-x_{1}$. Figure 3 presents the graphs obtained for $1-x_{1}=0.2$ and the various $K$. Graphs for other values of $1-x_{1}$ display the same general forms. Graphs and tables of $v(x)$ are available for all the following cases:

$$
\begin{aligned}
& 1-x_{1}=0.01 \text { with } K=0.001,0.005,0.01,0.05,0.1,0.5,1, \text { and } 5 . \\
& 1-x_{1}=0.2 \text { with } K=0.001,0.005,0.01,0.05,0.1,0.5,1, \text { and } 5 . \\
& 1-x_{1}=0.4 \text { with } K=0.005,0.01,0.05,0.1,0.5,1,5, \text { and } 10 . \\
& 1-x_{1}=0.6 \text { with } K=0.01,0.05,0.1,0.5,1,5, \text { and } 10 . \\
& 1-x_{1}=0.8 \text { with } K=0.05,0.1,0.5,1,5,10,50, \text { and } 100 \\
& 1-x_{1}=0.9 \text { with } K=0.1,0.5,1,5,10,50, \text { and } 100 .
\end{aligned}
$$

The experimental test case corresponded to $1-x_{1}=0.004970$ and $K=0.03713$.

Initial slopes ranged from -0.000296 (for $1-x_{1}=0.01$ and $K=0.001$ ) to -3.463 (for $1-x_{1}=0.8$ and $K=100$ ). Intervals of integration necessary to give 3 decimal accuracy ranged from 1.0 for the smaller $1-x_{1}$ and $K$, to 0.008 for the larger values. The effective value of $x \rightarrow \infty$, as defined above, ranged similarly from 173 to 1.25 . A reversal of the general trends was noted as $s$ became greater than unity.

The author is greatly indebted to Professor C. C. Gotlieb for advice in preparing this manuscript, and to Mrs. MARY BURGESS, who organized and carried through the production schedule for the floating-point program, and displayed much ingenuity in performing the interpolations. The chemical significance of the solutions is to be discussed in a separate paper [2]. The problem was submitted to the Computation Centre by the National Research Council of Canada.

BeATrice H. Worsley

Computation Centre

University of Toronto

Canada

${ }^{1}$ R. J. Cvetanović \& D. J. Leroy, "Contribution to the theory of diffusion flames," Canadian Jn. Chem., v. 29,1951 , p. 597-603.

${ }^{2}$ R. J. Cvetanović, to be published.

${ }^{3} \mathrm{~S}$. GiLl, "A process for the step-by-step integration of differential equations in an automatic digital computing machine," Cambridge Phil. Soc., Proc., v. 47, 1950, p. 96-108.

${ }^{4}$ B. H. WORSLEY, "Numerical representation in fixed-point computers," Computers and Automation, v. 4, No. 5, 1955, p. 10-13.

${ }^{5}$ In retrospect, it would appear that a MILNE-type formula, with programmed calculation of starting values, could have been more profitably employed. 